\title{
A CARLESON MEASURE THEOREM FOR BERGMAN SPACES
}

\author{
WILLIAM W. HASTINGS
}

ABSTRACT. Let $\mu$ be a finite, positive measure on $U^{n}$, the unit polydisc in $C^{n}$, and let $\sigma_{n}$ be $2 n$-dimensional Lebesgue volume measure on $U^{n}$. For $1 \leq p \leq q<\infty$ a necessary and sufficient condition on $\mu$ is given in order that $\left\{\int_{U^{n}} f^{q}(z) d \mu(z)\right\}^{1 / q} \leq C\left\{\int_{U^{n}} f^{p}(z) d \sigma_{n}(z)\right\}^{1 / p}$ for every positive $n$-subharmonic function $f$ on $U^{n}$.

A theorem of Carleson [1], [2] as generalized by Duren [3] characterizes those positive measures $\mu$ on $|z|<1$ for which the $H^{p}$ norm dominates the $L^{q}(\mu)$ norm of elements of $H^{p}$. The purpose of this note is to prove an analogous result with $H^{p}$ replaced by $A^{p}$, the Bergman space of functions $f$ which are analytic in $|z|<1$ and for which $\int_{0}^{1} \int_{0}^{2 \pi}\left|f\left(r e^{i \theta}\right)\right|^{p} r d \theta d r<\infty$. Actually, the result is more general in that it applies to positive $n$-subharmonic functions and positive measures on the unit polydisc in $C^{n}$. I wish to express my gratitude to Professor Allen Shields for suggesting this problem and guiding me to its solution. My thanks also go to the referee for outlining a correction to an error in my proof.

First, some notation and a definition. Let

$$
U^{n}=\left\{z=\left(z_{1}, \ldots, z_{n}\right) \in \mathbf{C}^{n}:\left|z_{j}\right|<1,1 \leq j \leq n\right\},
$$

and let $\sigma_{n}$ be $2 n$-dimensional Lebesgue volume measure restricted to $U^{n}$, normalized so that $U^{n}$ has measure one. Suppose that $f$ is upper semicontinuous on $U^{n}$. Then we say that $f$ is $n$-subharmonic provided that $f$ is subharmonic in each variable separately (cf. [5, p. 39]).

Theorem. Let $\mu$ be a finite, positive measure on $U^{n}$, and suppose $1 \leq$ $p \leq q<\infty$. Then there exists a constant $C>0$ such that

$$
\left\{\int_{U^{n}} f^{q(z) d \mu(z)}\right\}^{1 / q} \leq C\left\{\int_{U^{n}} f^{\left.p(z) d \sigma_{n}(z)\right\}^{1 / p}}\right.
$$

for every positive n-subharmonic function $f$ on $U^{n}$ if and only if there exists a constant $C^{\prime}>0$ such that

$$
\mu(S) \leq C^{\prime}\left(\prod_{j=1}^{n} \delta_{j}\right)^{2 q / p}
$$

for every set $S$ of the form

$$
S=\left\{z=\left(r_{1} e^{i \theta_{1}}, \ldots, r_{n} e^{i \theta} n\right): 1-\delta_{j} \leq r_{j}<1, \theta_{j}^{0} \leq \theta_{j} \leq \theta_{j}^{0}+\delta_{j}, 1 \leq j \leq n\right\} .
$$

Received by the editors April 23, 1974 and, in revised form, July 1, 1974. AMS (MOS) subject classifications (1970). Primary 30A78, 32A30, 46E 15.

Key words and phrases. Bergman spaces, $n$-subharmonic function, finite positive measure. 
Proof. Suppose that $0<p \leq q<\infty$, and suppose that (1) holds for every positive $n$-subharmonic function $f$ on $U^{n}$. Let $S$ be a set of the form (3). Let

$$
a_{j}=\left(1-\delta_{j}\right) \exp \left\{i\left(\theta_{j}^{0}+\delta_{j} / 2\right)\right\}, \quad 1 \leq j \leq n
$$

and set

$$
f(z)=\prod_{j=1}^{n}\left|1-\bar{a}_{j} z_{j}\right|^{-4 / p} .
$$

A geometric argument $[4$, p. 157] shows that in $S$,

$$
f(z) \geq c_{1} \prod_{j=1}^{n} \delta_{j}^{-4}
$$

Therefore,

$$
c_{1}^{q / p} \prod_{j=1}^{n} \delta_{j}^{-4 q / p} \mu(S) \leq \int f^{q} d \mu \leq C^{q}\left\{\int f^{p} d \sigma_{n}\right\}^{q / p} \leq C^{q} \prod_{j=1}^{n} \delta_{j}^{-2 q / p}
$$

and (2) holds with $C^{\prime}=c_{1}^{-q / p} C^{q}$.

Conversely, suppose that (2) holds for every set $S$ of the form (3). For $m=\left(m_{1}, \ldots, m_{n}\right) \in \mathbf{Z}^{n}$ and $k=\left(k_{1}, \ldots, k_{n}\right) \in \mathbf{Z}^{n}$ with $m_{j} \geq 0$ and $1 \leq k_{j}$ $\leq 2^{m j+4}, 1 \leq j \leq n$, set

$$
\begin{aligned}
T_{m k}=\left\{z=\left(r_{1} e^{i \theta_{1}}, \ldots, r_{n} e^{i \theta} n\right): 1-2^{-m_{j}} \leq r_{j}<1-2^{-m_{j}-1},\right. \\
\left.2 k_{j} \pi / 2^{m_{j}+4} \leq \theta_{j}<2\left(k_{j}+1\right) \pi / 2^{m_{j}+4}, 1 \leq j \leq n\right\},
\end{aligned}
$$

and set $z^{m k}=\left(z_{1}^{m k}, \ldots, z_{n}^{m k}\right)$, where

$$
z_{j}^{m k}=\left(1-2^{-m}\right) \exp \left\{2\left(k_{j}+1 / 2\right) \pi i / 2^{m_{j}+4}\right\}, \quad 1 \leq j \leq n .
$$

Note that

$\mu\left(T_{m k}\right) \leq C\left(\prod_{j=1}^{n} 2^{-m_{j}}\right)^{2 q / p} \quad$ and $\max _{z \in T_{m k}}\left|z_{j}-z_{j}^{m k}\right|<\frac{11}{16} 2^{-m_{j}}, \quad 1 \leq j \leq n$.

Now suppose that $f$ is positive and $n$-subharmonic in $U^{n}$. For $(6 / 8) 2^{-m_{j}}$ $\leq \rho_{j} \leq(7 / 8) 2^{-m_{j}}$ and $z \in T_{m k}$, repeated application of Harnack's inequality yields

$$
\begin{aligned}
f^{p}(z) \leq(2 \pi)^{-n} & \prod_{j=1}^{n}\left(\frac{\rho_{j}+\left|z_{j}-z_{j}^{m k}\right|}{\rho_{j}-\left|z_{j}-z_{j}^{m k}\right|}\right) \\
& \quad \int_{0}^{2 \pi} \cdots \int_{0}^{2 \pi} f^{p}\left(z_{1}^{m k}+\rho_{1} e^{i \theta_{1}}, \cdots, z_{n}^{m k}+\rho_{n} e^{i \theta_{n}}\right) d \theta_{1} \cdots d \theta_{n} \\
& \leq c_{1}(2 \pi)^{-n} \int_{0}^{2 \pi} \cdots \int_{0}^{2 \pi} f^{p}\left(z_{1}^{m k}+\rho_{1} e^{i \theta_{1}}, \ldots, z_{n}^{m k}+\rho_{n} e^{i \theta_{n}}\right) d \theta_{1} \cdots d \theta_{n}
\end{aligned}
$$

Hence, for $z \in T_{m k}$, 


$$
\begin{aligned}
f^{p}(z) & =c_{2}\left(\prod_{j=1}^{n} 4^{m_{j}}\right) \int_{(6 / 8) 2}^{(7 / 8) 2^{-m_{1}} \cdots \int_{(6 / 8) 2}^{-m_{n}}} \int^{(7 / 8) 2^{-m_{n}}}{ }^{p(z) \rho_{1} \cdots \rho_{n} d \rho_{1} \cdots d \rho_{n}} \\
& \leq c_{1} c_{2}\left(\prod_{j=1}^{n} 4^{m_{j}}\right) \int_{U_{m k}} f^{p} d \sigma_{n}
\end{aligned}
$$

where

$$
U_{m k}=\left\{z=\left(z_{1}, \ldots, z_{n}\right) \in \mathbf{C}^{n}:\left|z_{j}-z_{j}^{m k}\right| \leq(7 / 8) 2^{-m}, 1 \leq j \leq n\right\} .
$$

Since $z$ was arbitrary in $T_{m k}$, we have

$$
\begin{aligned}
\int_{U^{n}} f^{q} d \mu & =\sum_{m=\left(m_{1}, \ldots, m_{n}\right)} \sum_{k=\left(k_{1}, \ldots, k_{n}\right)} \int_{m_{m k} \geq 0} f^{q} d \mu \\
& \leq \sum_{m} \sum_{k} \mu\left(T_{m k}\right)\left\{c_{j} \leq 2^{m_{j}+4} c_{2}\left(\prod_{i=1}^{n} 4^{m^{2}}\right) \int_{U_{m k}} f^{p} d \sigma_{n}\right\}^{q / p} \\
& \leq c_{3} \sum_{m} \sum_{k}\left\{\int_{U_{m k}} f^{p} d \sigma_{n}\right\}^{q / p}
\end{aligned}
$$

Fix $m^{0}=\left(m_{1}^{0}, \ldots, m_{n}^{0}\right)$ and $k^{0}=\left(k_{1}^{0}, \ldots, k_{n}^{0}\right)$ with $m_{j}^{0} \geq 0$ and $1 \leq k_{j}^{0}$ $\leq 2^{m}{ }_{j}^{0}+4,1 \leq j \leq n$. We claim that $T_{m^{0} k^{0}}$ intersects $U_{m k}$ for at most $N=$ $(5.57)^{n}$ choices of the pair $(m, k)$. Assume first that $m_{j}^{0} \geq 1$. If

$$
z=\left(r_{1} e^{i \theta_{1}}, \ldots, r_{n} e^{i \theta} n\right) \in T_{m}{ }^{0}{ }_{k} 0
$$

then $1-2^{-m_{j}^{0}} \leq r_{j}<1-2^{-m_{j}^{0}-1}$, while if $z \in U_{m k}$, then $1-2^{-m_{j}+1}<r_{j} \leq$ $1-2^{-m_{j}-3}$. Hence, if $z \in T_{m}{ }_{k} 0 \cap U_{m k}$, then combination of the two inequalities shows that $m_{j}^{0}-3 \leq m_{j}<m_{j}^{0}+2$. Then $m_{j}$ can be one of at most five different values. Similarly, if $z \in T_{m^{0}{ }_{k}}$, then we may assume that

$$
2 k_{j}^{0} \pi / 2^{m_{j}^{0}+4} \leq \theta_{j}<2\left(k_{j}^{0}+1\right) \pi / 2^{m_{j}^{0}+4} \text {. }
$$

A little geometry shows that if $z \in U_{m k}$, then

$$
\frac{2\left(k_{j}+1 / 2\right) \pi}{2^{m_{j}+4}}-\frac{7 \pi}{8} 2^{-m_{j}} \leq \theta_{j}+2 l \pi \leq \frac{2\left(k_{j}+1 / 2\right) \pi}{2^{m_{j}+4}}+\frac{7 \pi}{8} 2^{-m_{j}}
$$

where $l$ is either 0,1 or -1 . Combination of the se two inequalities yields

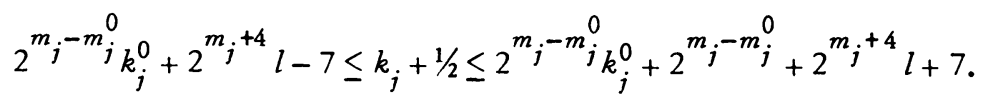


Hence, if $z \in T_{m{ }^{0} k^{0}} \cap U_{m k}$, then $k_{j}$ can be one of at most $3\left(15+2^{m_{j}^{-m_{j}}}{ }_{j}\right)$ $\leq 57$ different values. (The factor of 3 reflects the three possible values of l.) This establishes the claim for $m_{j}^{0} \geq 1$, but if $m_{j}^{0}=0$ for any $j$ in the above counting procedure, then we would have even fewer intersections.

Therefore,

$$
\begin{aligned}
& \sum_{m=\left(m_{1}, \ldots, m_{n}\right)} \sum_{k=\left(k_{1}, \ldots, k_{n}\right)}\left\{\int_{U_{m k}} f^{p} d \sigma_{n}\right\}^{q / p} \leq\left\{\sum_{m, k} \int_{U_{m k}} f^{p} d \sigma_{n}\right\}^{q / p} \\
& m_{j} \geq 0 \quad 1 \leq k_{j} \leq 2^{m_{j}+4} \\
& =\left\{\sum_{m, k} \sum_{m^{0}, k} \int_{T_{m}{ }^{0} 0^{0}} \cap U_{m k} f^{p} d \sigma_{n}\right\}^{q / p} \\
& =\left\{\sum_{m^{0}, k} \sum_{m, k} \int_{T_{m} 0_{k} 0^{\cap U_{m k}}} f^{p} d \sigma_{n}\right\}^{q / p} \\
& \leq\left\{N \sum_{m^{0}, k} \int_{T m^{0} k^{0}} f^{p} d \sigma_{n}\right\}^{q / p}=N^{q / p}\left\{\int_{U^{n}} f^{p} d \sigma_{n}\right\}^{q / p},
\end{aligned}
$$

and the proof is complete.

Remark. The Theorem also holds for $0<p \leq q<\infty$ if we require that $f=|g|$, where $g$ is holomorphic in $U^{n}$. In this case $f^{p}$ is $n$-subharmonic, and so the proof is the same.

As in [3] two inequalities follow immediately.

Corollary. For a positive subharmonic function $f$ on $|z|<1$ and for $1 \leq p \leq q<\infty$

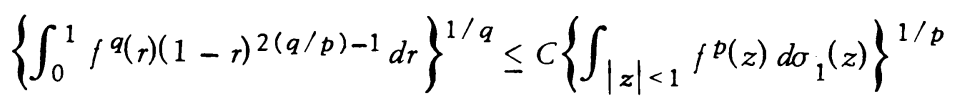

and

$$
\left\{\int_{0}^{1}(1-r)^{2(q / p)-2} \int_{0}^{2 \pi} f^{q\left(r e^{i \theta}\right)} d \theta d r\right\}^{1 / q} \leq C^{\prime}\left\{\int_{|z|<1} f^{\left.p(z) d \sigma_{1}(z)\right\}^{1 / p}}\right.
$$

where the constants $C$ and $C^{\prime}$ may be chosen independently of $f$.

For another application, suppose $\left\{z_{j}\right\}_{j=1}^{\infty}$ is a sequence of distinct points in $|z|<1$. Let $\mu$ be the point measure defined by $\mu\left\{z_{i}\right\}=\left(1-\left|z_{j}\right|^{2}\right)^{2}, j \geq 1$, and $\mu\left(U \backslash\left\{z_{j}\right\}_{j=1}^{\infty}\right)=0$. For $f \in A^{p}(p>0)$ let $T_{p} f$ be the sequence $\left\{f\left(z_{j}\right)\left(1-\left|z_{j}\right|^{2}\right) 2 / p\right\}_{j=1}^{\infty}$.

Corollary. For $0<p<\infty, T_{p}\left(A^{p}\right) \subset l^{p}$ if and only if $\mu(S) \leq c \delta_{1}^{2}$ for every set $S$ of the form (3) with $n=1$. 


\section{REFERENCES}

1. L. Carleson, An interpolation problem for bounded analytic functions, Amer. J. Math. 80 (1958), 921-930. MR 22 \#8129.

2. - Interpolations by bounded analytic functions and the corona problem, Ann. of Math. (2) 76 (1962), 547-559. MR 25 \#5186.

3. P. L. Duren, Extension of a theorem of Carleson, Bull. Amer. Math. Soc. 75 (1969), 143-146. MR 39 \#2989.

4. - Theory of $H^{p}$ spaces, Pure and Appl. Math., vol. 38, Academic Press, New York, 1970. MR 42 \#3552.

5. Walter Rudin, Function theory in polydiscs, Benjamin, New York, 1969. MR 41 \#501.

DEPARTMENT OF MATHEMATICS, UNIVERSITY OF MICHIGAN, ANN ARBOR, MICHIGAN 48104

Current address: Department of Mathematics, Fordham University, Bronx, New York 10458 